Psychosis proneness - a basic personality trait complementing the HEXACO model

\title{
Proneness to psychotic-like experiences as a basic personality trait complementing the HEXACO model - A preregistered cross-national study
}

Goran Knežević ${ }^{1}$, Ljiljana B. Lazarevic ${ }^{2}$, Michael Bosnjak ${ }^{3}$, \& Johannes Keller ${ }^{4}$

1) Department of Psychology, University of Belgrade, Serbia

2) Institute of Psychology, University of Belgrade, Serbia

3) ZPID - Leibniz Institute for Psychology Information and University of Trier, Germany

4) Department of Psychology \& Education, Ulm University, Germany

Address correspondence to:

Goran Knežević,

Faculty of Philosophy, University of Belgrade

Belgrade, Serbia

E-mail: gknezevi@f.bg.ac.rs

\section{Acknowledgment}

We would like to thank PsychLab, a service of the Leibniz Institute for Psychology Information (ZPID) in Trier, Germany, for founding data collection of the present study.

\section{Disclosures}

The study design and analyses were preregistered. The preregistration data, code to reproduce our analyses, and materials used in the study are available at https://osf.io/x6tcr/?view_only=9dec13e973fe4ab8b131e265c1380db5. We report how we determined our sample size, all data exclusions, all manipulations, and all measures in the study. Data were collected following the Declaration of Helsinki. The study was approved by the IRB of the Department of psychology, Faculty of Philosophy, University of Belgrade, Serbia, Protocol \#2020-70.

PLEASE NOTE THAT THIS IS A PRE-PRINT, WHICH HAS BEEN SUBMITTED TO A JOURNAL. WHILE THIS VERSION OF THE DOCUMENT IS THE MOST COMPLETE AND MOST RECENT, IT SHOULD BE REGARDED AS A WORK IN PROGRESS.

WE URGE ANY PARTIES INTERESTED IN THIS WORK TO CONTACT THE LEAD AUTHOR (LISTED ABOVE) FOR FURTHER DETAILS OR UPDATES. 
Psychosis proneness - a basic personality trait complementing the HEXACO model

\section{Abstract}

The goal of the study is to investigate the relationship between the HEXACO personality model and Disintegration - representing a broad spectrum of psychotic-like experiences and behavioral tendencies that are reconceptualized as a personality trait. In this pre-registered study, we predicted that the Disintegration factor would separate from HEXACO.

The replicability of the factorial structures of HEXACO and Disintegration subcomponents are investigated across the three national samples (X, Y, and Z), matched on key socio-demographic variables. Exploratory Structure Equation Modeling (ESEM) is used to study the invariance of the hypothesized seven-factor structure.

Support for the metric invariance of the seven-factor structure based on HEXACO and Disintegration subcomponents/facets across the three nations was found. The disintegration factor lied clearly outside the HEXACO personality space with each of its nine subcomponents. The disintegration factor appeared to be the most robust among the seven across the samples and units of measurement (facets and items). A broad spectrum of psychotic-like experiences/behavioral tendencies relevant in understanding and explaining many aspects of everyday and long-term (mal)adaptations - as expected - is not captured by the HEXACO model.

Keywords: HEXACO personality model; Disintegration; Proneness to psychotic-like experiences/behaviors; Seven-factor personality model; Cross-national metric invariance; 
Psychosis proneness - a basic personality trait complementing the HEXACO model

\section{Introduction}

The HEXACO personality model, postulating the existence of the six basic personality traits - Honesty/Humility (H), Emotionality (E), Extraversion (X), Agreeableness (A), Conscientiousness, and Openness (O) - gained substantial empirical support (see Thielmann et al., 2019). Some prominent advocates of the lexical approach to personality claim that "evidence to date indicates that the replicability of the six-factor structure (roughly, Big Five + Honesty) across languages probably exceeds that for the Big Five" (Saucier, 2008, p. 41). However, there is growing evidence that the proneness to psychotic-like experiences is represented neither in the HEXACO (e g. Ashton \& Lee, 2012; 2020), nor the other most influential contemporary personality models, such as Big Five (Asthon \& Lee 2020; Knežević et al., 2016, 2017; Watson et al., 2008), Eysenck's PEN model (Knežević et al., 2019), or Cloninger's personality model (Lazarevic et al., 2016).

At the outset, it is certainly relevant to address the basic question of why psychosis proneness should be represented in a comprehensive model of basic personality traits? From an empirical perspective, it appears that there is solid evidence indicating that psychosis proneness satisfies the criteria defined by Eysenck (1977) or Momirovic (1998) to consider it a basic personality trait. That is, psychotic-like phenomena showed a dimensional quality (e.g., Kwapil \& Barrantes-Vidal, 2014), repeatedly and consistently showed factorial coherence and separation from other personality factors (Asthon \& Lee 2020; Knežević et al., 2017; Watson et al., 2008), there is evidence documenting temporal stability (e.g., Sanislow et al., 2009), cross-national factorial stability (e.g. Fonseca-Pedrero, 2017), evidence on genetic variance that is similar to the genetic variance found for other personality factors (e.g., Ronald, 2015), convincing elaborations on the underlying biological factors (e.g., Philips \& Silverstein, 2003), and relevance concerning 
Psychosis proneness - a basic personality trait complementing the HEXACO model

many aspects of everyday behavior and psychological mal(functioning), such as psychotic and non-psychotic psychopathology (e.g., Rössler et al., 2011), spiritual experiences (e.g., Jackson,1997), paranormal beliefs (e.g., Goulding, 2005), some forms of creativity (Nettle, 2006), socio-political attitudes, worldviews, and thinking styles (Knežević \& Keller, 2020), various aspects of emotional processing (Kerns, 2006), some aspects of militant extremism (Medjedović \& Knežević, 2019), or health-related behaviors during the COVID-19 pandemic (Lazarevic et al., 2021).

There are numerous reasons which probably contributed to the fact that the tendency to psychotic-like experiences/behaviors has not been found or, at least, has not been consistently ${ }^{1}$ found in lexical studies and these reasons were already discussed elsewhere (for example, Knežević et al., 2016, 2017 or Watson et al., 2008). Instead of reiterating them here, we refer to a recent statement by proponents of the lexical approach - Ashton and Lee. Summarizing available empirical evidence, they stated that "researchers interested in integrating the domains of normal and abnormal personality are advised to adopt a model consisting of six HEXACO-like dimensions plus a dimension of psychotic tendency" (Ashton \& Lee, 2020, p. 1). This coincides well with the major claim by Knežević et al. (2017), who conceptualized the domain of psychoticlike experiences as a personality trait with nine subdimensions and labeled it Disintegration. The Disintegration factor was empirically established as separate from the Five-Factor Model (FFM) of personality. This finding was replicated across informant types, samples, units of analyses, and the way items of Disintegration were presented to the subjects.

1 It can be argued that something that might be qualified as psychotic-like was found in the lexical seven-factor solutions (which include a so-called "Negative Valence" factor) based on emic studies of Hebrew and Filipino languages (Saucier, 2003). In Saucier's factor analysis of 60 marker adjectives of seven factors (basically HEXACO + Negative Valence) the descriptors with the highest loadings on the Negative Valence factor were: Insane, Crazy, Good-for-nothing, Corrupt, Evil, Weird, and Stupid. 
Psychosis proneness - a basic personality trait complementing the HEXACO model

\section{The characteristics of the Disintegration factor}

One of the important qualities of the Disintegration (D) factor is that it has been established as a comprehensive representation of psychotic-like experiences, encompassing a broad spectrum of phenomena. In the first phase of the empirical work, around one thousand indicators of various psychotic-like phenomena were considered, based on the most influential models of psychosis proneness, including items based on the models proposed by Chapmans and Claridge (e.g., Claridge et al., 1996). In a series of factor analyses of these items, nine subdimensions which strongly converged to a higher-order factor were identified (Knežević et al., 2017): General Executive/Cognitive Impairment (GE/CI), Perceptual Distortions (PD), Enhanced Awareness (EA), Apathy/Depression (A/D), Paranoia (PAR), Mania (MAN), Flattened Affect (FA), Somatoform Dysregulation (SOD), and Magical Thinking (MT). It is important to note that although broad, the D construct is distinct from the construct introduced as General Factor of Psychopathology, recently suggested by Caspi et al. (2014). Namely, the D does not include the broad domains of anxiety-related (internalization), aggressive and antisocial (externalization), disinhibitory, and anhedonic phenomena (both social and physical).

The phenomena captured by the GE/CI factor represent a broad spectrum of executive and memory impairment indices "evident on a background of a generalized cognitive deficit" (Reichenberg \& Harvey, 2007, p. 833), such as dysregulation of attention, planning, memory, concentration, speech comprehension and production, motor control, coordination between intention and motor outputs, but also phenomena such as blackouts, absent-mindedness, hypoawareness of one's behavior, obsessions and compulsions. This factor is found in the majority of psychosis proneness models, predominantly labeled as disorganization (e.g., Kwapil \& BarrantesVidal, 2014). The central contents of PD are phenomena of depersonalization, derealization 
Psychosis proneness - a basic personality trait complementing the HEXACO model

(including experiencing multiple identities), as well as Schneiderian first-rank symptoms. This subdimension - representing the core content of D - exists in all models of psychosis proneness, either as a separate substructure (e.g., Krueger et al., 2012) or as part of the Positive Symptom factor (e.g., Kwapil \& Barrantes-Vidal, 2014). The EA subdimension comprises contents such as heightened awareness, synesthesia, responsiveness to engaging stimuli, increased cognition, dissociative involvement related to sounds/music, and vivid reminiscence. Phenomena such as chronic fatigue, feelings of uselessness, hopelessness, helplessness, and suicidal ideation are part of the A/D subdimension. Several models of psychotic-like phenomena described depression as part of the psychotic symptomatology, such as the five-factor model by Lindenmayer et al. (2004). Phenomena such as suspiciousness, distrustfulness, oversensitivity, paranoid resentment, ideas of persecution, and conspiracy belong to the PAR subdomain. In several models of psychosis proneness, PAR has been postulated or extracted as a subdimension separate from the Positive Symptom factor (e.g., Stuart et al., 1999) or an aspect of it (e.g., Lenzenweger \& Dworkin, 1996). The subdimension MAN is characterized by overactivity, agitation, recklessness, extreme optimism, elevated mood, inflated self-esteem, and excessive grandiosity. Previous studies found a similar factor that has been included in influential models of psychosis proneness, such as Serretti and Olgiati's (2004) five-factor model. The extraction of this factor but also A/D and FA further demonstrate that emotional dysregulation (characterized by the cyclothymic fluctuation of extreme emotional states, both with positive and negative valence, temporarily replaced by the periods of blunted affect) is a component of psychosis-proneness. The FA subdimension is characterized by the emotional indifference towards the self and others, emotional numbing, lack of future planning, and superficiality, not outward expression of emotions in face or voice. Despite being predominantly connected to the diminished emotional 
Psychosis proneness - a basic personality trait complementing the HEXACO model

expressiveness, flattened affect symptom severity was shown to be related also to the impairment in discriminating and experiencing valence-specific emotions (Gur et al., 2006). The factor SOD comprises experiences of organ malfunction and damage, severe forms of sensory and motor conversions, insensitivity to pain, and general body numbing. This factor does not include body image distortions - which are at the core of the PD subdomain. The contents of the MT subdomain are feeling telepathic, having energetic connections with others, illogical thinking, belief in the afterlife and reincarnation, magical influences, and horoscopes. Extraction of this factor is consistent with empirical evidence documenting MT as one of the key features of the proneness to schizophrenia (Goulding, 2005). These phenomena are usually located in the subdomain of positive symptoms (see Kwapil \& Barrantes-Vidal, 2014).

\section{Goals of the study}

Although several studies already supported the distinctiveness of HEXACO traits and psychotic-like experiences (such as Ashton \& Lee, 2012, 2020; Ashton et al., 2012; Medjedovic, 2014) this is, to the best of our knowledge, the first one to test this assumption cross-culturally while using the construct capturing psychosis proneness broadly (i.e., not restricting it to positive symptoms). Therefore, the main research question addressed in the current work is whether the D factor separates from the HEXACO factors across three different cultural groups, namely residents of the $X, Y$, and $Z$.

Having in mind Ashton and Lee's earlier position (2012) - according to which the domain of psychosis proneness was hardly anything beyond perceptual distortions, phenomena of dichotomous nature, exceptionally rare in the general population, and therefore not belonging to the realm of personality - it would be of interest to investigate whether subscales of this broadly conceptualized proneness to psychotic-like experiences "go together" and form a factor distinct 
Psychosis proneness - a basic personality trait complementing the HEXACO model

from the HEXACO factors. Ashton and Lee (2020) abandoned their earlier position - given that they recently accepted the necessity to include the dimension of "psychotic tendencies" in a comprehensive description of personality variations. Our findings might contribute to the precise charting of the contents and the boundaries of this domain.

The following pre-registered hypotheses ${ }^{2}$ were tested:

H1. Disintegration facets as conceptualized by Knežević et al. (2017) form a D factor, separate from the six HEXACO factors. We expect that the seven-factor personality structure extracted using Exploratory Structural Equation Modelling (ESEM) shows adequate GoF (Goodness-of-Fit) indices.

H2. The seven-factor structure encompassing the six HEXACO factors and one separate D factor will be a) robust across the three national samples, b) robust across the units of analysis (subscales and items) on which these factors are based. Specifically, we expect multi-group ESEM to reveal metric invariance of the solution (H2a). Moreover, we expect that comparing the seven-factor solution extracted at the level of HEXACO + D facets and the seven-factor solution extracted at the level of HEXACO + D items, the robustness of D revealed through the correlations between the D factor extracted from scales and items is similar to the robustness of the HEXACO factors $(\mathrm{H} 2 \mathrm{~b})$.

\section{Method}

\section{Sample and procedure}

Taking into account that not only commonality of the variables can influence the stability of factor solutions (MacCallum et al., 1999), but also sample size, in the sense that greater

2 https://osf.io/x6tcr/?view_only=9dec13e973fe4ab8b131e265c1380db5 
Psychosis proneness - a basic personality trait complementing the HEXACO model

stability (smaller standard deviations of loadings across repeated samples) and more accurate recovery of the population loadings can be obtained in larger samples (Browne, 1968), focused on adequate sample size. The most frequent recommendations regarding the variable-tosubject ratio are between 1:5 (e.g., Gorsuch, 1983) to 1:10 (e.g., Everitt, 1975). As we have 33 facets in the factor analysis (24 HEXACO + 9 DELTA), the 1:10 variable-to-subject ratio can be achieved with 340 respondents. To perform factor analysis at the item level, the same ratio in a factor analysis on the overall sample (given that the number of HEXACO + DELTA items is $96^{3}$ $+20=116$ ) can be retained with around 1100 respondents. Therefore, in each of the samples (countries), we planned to collect at least 360 respondents, which results in about 1100 respondents altogether.

The seven-factor personality structure was investigated with data from three community samples representing three nations - the X, Y, and Z. Our decision to collect data in these three countries was based on several criteria. First, an important factor was the availability of samples representative of the population. Second, claiming the robustness of the seven-factor solution requires collecting data in countries differing in characteristics that might be reflected in personality scores (at least to some extent). The selected countries differ concerning language, religion, and historico-cultural tradition. We also inspected some objective parameters that might be related to scores on personality inventories, such as beliefs, values, and motivation systems (Hofstede's dimensions of national culture, Hofstede et al., 2010) and WEIRDness scores (Henrich et al., 2010). On all criteria we analyzed, X differs from both Y and the Z. Still, there are several other indices on which the selected countries significantly differ from each other. Those are Hofstede's Collectivism - Individualism, Uncertainty Avoidance, and Indulgence dimensions

3 Following the approach of Ashton and Lee (2020), we decided not to include the Altruism facet in the factor analyses. 
Psychosis proneness - a basic personality trait complementing the HEXACO model

(details can be found at https://www.hofstede-insights.com/country-comparison/XXX).

Furthermore, the selected countries differ concerning WEIRDness scores. In the present case, we used the scores obtained as part of the Many Labs2 project (Klein et al., 2018): The three selected countries differ substantially on the dimension Industrialization $(\mathrm{Y}=.73, \mathrm{Z}=.40, \mathrm{X}=.04)$.

Therefore, we advocate that the inclusion of community samples from the three countries in the design of the study enables a certain level of cross-cultural diversity to investigate the robustness of the hypothesized seven-factor structure.

Data in $\mathrm{X}$ were collected before pre-registration, while data in $\mathrm{Y}$ and the $\mathrm{Z}$ were collected following pre-registration (see pre-registration document mentioned in footnote 2). X data were collected as part of the process of standardization of the WAIS-IV intelligence test on a normative sample representative of the general population. Samples in the $\mathrm{Z}$ and $\mathrm{Y}$ were planned to match the $\mathrm{X}$ sample regarding gender, age, and education level. According to age, sample quotas were the following: $18-20$ years $(13 \%), 21-30$ years $(20 \%), 31-40$ years $(17 \%), 41-50$ years $(14 \%), 51-$ 60 years $(16 \%), 61-75$ years $(20 \%)$. Regarding educational level, the sample was planned as follows: 20\% - 8 years (elementary school and lower), 60\% - 9-12 years (secondary school, vocational), and $20 \%$ - more than 12 years of education (college, university, Ph.D.).

The $\mathrm{X}$ sample comprised $\mathrm{N}=368$ respondents, $55 \%$ female, mean age 42.74 years ( $\mathrm{SD}=17.93)$. Y sample consisted of $\mathrm{N}=374$ respondents, $50 \%$ female, mean age 42.93 ( $\mathrm{SD}=16.84)$. The $\mathrm{Z}$ sample consisted of $\mathrm{N}=363$ respondents, $54 \%$ female, mean age 43.67 $(\mathrm{SD}=15.51)$. In $\mathrm{X}$, data were collected by approaching people in person, while in the $\mathrm{Y}$ and $\mathrm{Z}$ data were collected via Talk Online Panel, a German-based portal, using Unipark software. The fact that only approximately $60 \%$ of participants in $\mathrm{X}$ who were recruited as part of the sample to establish normative scores for the WAIS-IV volunteered to continue filling in additional 
Psychosis proneness - a basic personality trait complementing the HEXACO model

inventories resulted in males and those with lower educational levels being slightly underrepresented regarding the population quotas. The likelihood that this process of selfselection in the $\mathrm{X}$ sample was related to personality might have influenced the representativeness of the personality scores for the general population in $\mathrm{X}$. In the $\mathrm{Z}$ and $\mathrm{Y}$, there were no missing values, while there were $0.7 \%$ missing values in the $\mathrm{X}$ database.

Lee and Ashton (2018) suggested a procedure to screen out participants who respond in a meaningful way. It sets a threshold for eliminating participants whose raw responses (i.e., before recoding of reverse-keyed items) show very little variability (SD below 0.7 across the 100 items). The rationale for such a procedure is the following: giving the same or nearly the same response across all items (non-keyed and reverse keyed) inflates variance across participants that is attributable to elevation of responses and deflates variance due to the actual personality traits. To avoid this, the procedure was applied to screen out our participants. It led to an exclusion of 94 participants: 24 from the $\mathrm{Z}$ sample, 69 from the $\mathrm{Y}$, and 1 from the $\mathrm{X}$ sample, leaving 1011 participants altogether.

\section{Instruments and Variables}

HEXACO personality traits. The HEXACO Personality Inventory-Revised (HEXACO PI-R; Lee \& Ashton, 2018) consists of 100 items. The instrument assesses six aforementioned basic personality traits and 25 lower-level dimensions (four facets per domain and one interstitial lower level trait, i.e., Altruism). We used the Y (Ashton \& Lee, 2007), Z (Lee \& Ashton, 2018), and X form (Medjedović et al., 2017), respectively. As already noted, in the factorial analyses reported below, the Altruism facet was not included.

Disintegration. To assess the D trait, we used a 20-item DELTA scale (posted at the site given in footnote 2). The questionnaire allows for the calculation of the overall $\mathrm{D}$ score and 9 
Psychosis proneness - a basic personality trait complementing the HEXACO model

aforementioned facet scores. The GC/EI and PD facets consist of three, and all other facets of two items. The $X$ form of the DELTA questionnaire (Knežević et al., 2017) was translated into German and English following the translation process of the Psychological Science Accelerator ${ }^{4}$ (Moshontz et al., 2018). Both measures have a 5-point Likert-type response scale ranging from 1 (strongly disagree) to 5 (strongly agree)

The 20-item form of the instrument (Knežević et al., 2017) was created using an Ant Colony Optimization (ACO) algorithm, a well-established procedure for conducting automated searches. The algorithm mimics the behavior of real ants by establishing the shortest route from their colony to sources of food by following the trail repeatedly marked by other ants via pheromone. The usefulness of ACO has already been demonstrated in developing short questionnaire forms (Olaru et al., 2015). The optimization criterion that we used was CFI, one of the important GoF indices in SEM, built on the comparison between noncentrality parameters of chi-square discrepancy distributions of the tested model and the basic, more restricted model, usually, a model assuming no covariances between variables (Bentler, 1990). This version of the DELTA questionnaire ${ }^{5}$ is ideally suited for this study because the number of items (20) is comparable to the number of HEXACO domain items (16). We also registered sociodemographic data: gender (coded with three categories: male, female, other), age in years, and educational level (coded with three categories: 1 - 8 years [elementary school and lower], $9-12$ [secondary school, vocational] and more than 12 years of education [college, university, Ph.D.]).

\section{Analytical strategy}

\footnotetext{
${ }^{4}$ https://psysciacc.org/translation-process/

${ }^{5}$ Several DELTA versions are available: 282 -item, 110-item, 50-item, and 10-item versions. The second and third version contain reverse-keyed items. All can be obtained upon requests.
} 
Psychosis proneness - a basic personality trait complementing the HEXACO model

To study the robustness of the seven-factor structure across the samples, multigroup ESEM (Exploratory Structural Equation Modeling, Marsh, et al., 2010) of 33 variables (24 HEXACO and 9 DELTA facets) was employed. We tested the configural, metric, and scalar invariance of the seven-factor model. The configural model imposes the least number of restrictions: It assumes invariance only concerning the number of factors. The metric model is more restrictive: It assumes not only the same number of factors across groups but also invariance of the factor loadings. However, in parallel to the configural one, it allows for intercepts and residuals of variables to be different and assumes factor means to be fixed at zero in all groups. Finally, the scalar model - the most restrictive one - assumes invariance of the number of factors, their loadings, and intercepts, while residuals are free for estimation. Factor means are fixed at zero in one group and free for estimation in all others. As in the metric model, factor variances are fixed to 1 in one group and free for estimation in the other groups. In all models, the factors are GEOMIN (oblique) rotated and correlations between them are free for estimation in each group. The MLR estimator - robust to the non-normality of variables' distributions - was used.

To evaluate the goodness-of-fit (GoF) of the model, several indices assessing misspecifications in both the structural (Standardized Root Mean Square Residual, SRMR) and the measurement aspects of a model (Root Mean Square Error of Approximation, RMSEA, and Comparative Fit Index, CFI) were examined. Hu and Bentler (1999) suggested that RMSEA should be less than 0.06 , CFI should be greater than 0.95 (although values from 0.90 to 0.95 can be considered acceptable according to Marsh et al., 2010), and SRMR should be less than 0.08. To compare models - instead of relying on the $\chi^{2}$ test statistic, which is seriously inflated for models involving a large number of variables (e.g., Herzog \& Boomsma, 2009) - we will rely on the differences between the models in the RMSEA and CFI. It has been suggested that $\Delta$ CFI $<.01$ 
Psychosis proneness - a basic personality trait complementing the HEXACO model

and $\triangle$ RMSEA $<.015$ imply that two models are not substantially different (e.g. Chen, 2007). The difference $(\Delta)$ in RMSEA will be crucial in deciding on the differences in the fit of models, having in mind that this specific GoF indicator incorporates a penalty for low parsimony of models such as ours, including a large number of parameters.

Furthermore, to study the robustness of the extracted factors across the units of analysis, factor scores based on subscales were compared with those based on items. Correlations above .90 between the factors based on subscales and items of a multifactorial inventory should be taken as an indicator of their reproducibility across units of analysis. There are many reasons why one should not expect such a high correspondence between the factors extracted on facets and items of a multidimensional (multifactorial) inventory. Facet scales of a personality inventory are mostly conceptual constructs not based on a bottom-up factorial approach. Thus, the choice of items to measure them is based on the local optimizations, i.e., those related to maximization of internal consistency of a particular subscale, its face and internal construct validity, or minimization of its correlations with other subscales belonging to the same domain. However, it can turn out that these items, working reasonably well within subscales, can reveal a different pattern if the factorial analysis is based directly on them. For example, inversely keyed items aligning well with the items forming a scale that represent one domain can turn out to be primary indicators of a different factor/construct if factor analysis is based on items instead of subscales (for example, a majority of the inversely keyed Agreeableness items in the NEO PI-R turn out to be primary indicators of Extraversion, Knežević et al., 2017). Although there are reasons why even some well-established factors look pretty different if they are based on items instead of subscales, if they correspond, it would be strong evidence for the conceptual soundness of the measures of such factors. In other words, this kind of analysis has less falsifying power (it cannot be 
Psychosis proneness - a basic personality trait complementing the HEXACO model

unequivocally concluded that disposition does not exist if the correlation between the corresponding factors based on its subscales and its items is low) than confirmatory (if there is a correspondence between the factors based on subscales and items above .90 this indicates the correct classification of the items into scales, i.e., better instrument design, and likely better conceptual understanding of a construct at the level of subdimensions). To obtain a stable sevenfactor solution at the level of items, the ESEM was computed on the overall sample (thus preserving the favorable number of variables/number of respondents ratio 10:1). These factor scores were then correlated with the factor scores of the seven multi-group ESEM factors extracted on subscales.

The robustness of the D factor is also tested by factorizing DELTA items first with nonkeyed and then, reverse-keyed HEXACO items. This is an important test of whether the separation of the D factor will be equally robust in the context of HEXACO items that are oriented toward healthy or maladaptive poles of personality traits. To have only items oriented toward the maladaptive pole of personality functioning make the items of HEXACO similar to what instruments such as PID-5 measure - maladaptive personality variations. If maladaptive poles of personality functioning create a context in which DELTA factor would not appear or would be severely distorted from what the D model suggests DELTA factors extracted in the contexts of ESEM with reverse-keyed and non-reverse-keyed HEXACO items would not have correlations above .90 .

Having in mind that the DELTA scale does not contain reverse-keyed items, it is likely that acquiescent responding accounts for a part of its variance. For this reason, the robustness of the seven-factor structure was tested when the tendency to acquiescent responding was taken into account. Given that HEXACO contains an equal number of keyed and non-keyed items, the 
Psychosis proneness - a basic personality trait complementing the HEXACO model

acquiescent score is calculated from it, then partialled out from each of the DELTA facets, before conducting ESEM based on HEXACO facets and these residualized DELTA facets.

ESEM analyses were performed in Mplus version 7 software (Muthén \& Muthén, 1998-

2012). All other analyses were completed in the SPSS package, Version 21.0.0.1.

\section{Results}

Descriptive indices for domains and facets across countries are displayed in Table 1. Despite its multicomponent nature and broadness of the behavioral domain, the reliability of the total D score is the largest among the domain scores - indicating thus the coherence of the central construct. Although the average scores of $\mathrm{D}$ and its subcomponents are lower than the average scores on HEXACO scales and subscales, the level of skewness of their distributions is far from indicating exceptional rareness of what might be considered symptoms that reflect the discrete nature of mental illnesses.

Correlations between HEXACO and DELTA facets and domains are presented in Appendix 1 in the Supplemental material at the site given in footnote 2. Results of the multigroup ESEM analyses support both the configural and metric but not scalar invariance of the seven-factor structure across the three nations (Table 2). We allowed for two error covariances to be estimated, the first, between Greed Avoidance and Modesty, and the second, between Sociability and Social Boldness. These two error covariances were found to be strong and consistent across the three samples and we concluded that they might be informative regarding certain aspects of the HEXACO model, even though this was detected post hoc. The differences in RMSEA between the configural and metric model are far below the suggested value of .015 to conclude that the configural (less restricted) model is more adequate than the metric (more restricted) model. It seems, therefore, that the loss in a fit of the more restricted (metric) model is 
Psychosis proneness - a basic personality trait complementing the HEXACO model

not so massive to decide that this more parsimonious model should be discarded. The most important finding was that the nine subscales postulated to constitute D were found to form a strong and coherent factor separate from the remaining six HEXACO factors in each of the three nations.

Table 1 about here

The metric invariance of the seven-factor structure assumes non-invariance of the intercepts of the facets. This implies that facets - despite having the same loadings on the factors across the nations - have different means, so the same levels of the latent variables are based on different facet level configurations. The fact that participants from the three samples had a different probability of responding with a certain score at the facet level despite having the same level of the latent trait represents evidence pointing at differential cross-cultural functioning of the facet levels. To investigate whether differential subscale functioning characterizes the D domain more than other personality domains, we tested to what extent partial intercept constraints decreased model fit. As can be seen in Table 2, constraining intercepts of D facets (Model 4d) caused a decrease of the overall model fit that is comparable to the decrease when the intercepts of the facets of the other HEXACO domains were constrained. This finding is of relevance, having in mind that the number of $\mathrm{D}$ facets is more than twice as large as the number of facets within HEXACO domains.

\section{Table 2 about here}

As depicted in Table 3, all factors are defined as expected. Somewhat problematic were unusually small loading of Modesty on $\mathrm{H}$, and the primary loading of Anxiety on X. The six- and five-factor solutions were not supported by our data, as revealed by the GoFs (Table 2). Of notice 
Psychosis proneness - a basic personality trait complementing the HEXACO model

is that it was not the D factor that collapsed in these solutions: in the six-factor solution $\mathrm{H}$ and $\mathrm{A}$ tend to fuse into one factor, while in the five-factor $\mathrm{O}$ and $\mathrm{C}$ coalesced together.

The seven-factor solution remained almost untouched after removing the acquiescent responding from DELTA facets (unstandardized residuals of these scores were used after each of them being regressed on the acquiescence score based on HEXACO items). This ESEM analysis is presented in Table 4 in the Supplemental material (the site is given in footnote 2).

Table 3 about here

Correlations between the ESEM factors varied across the nations/samples, but they were mostly below .30 (Table 3). Although some correlations were found to be even slightly above .40 , none of them was cross-nationally stable (or large) to the extent that it would render the fundamental separateness of the seven factors questionable. Of note, the correlations between D and $\mathrm{O}$ factors ranged between -.04 and .09 .

As expected, all ESEM factors had the highest correlations with their counterparts extracted on items (Table 5 in the Supplemental material). However, the correlations above .90 (all three) were registered in the case of all traits except $\mathrm{H}$ and $\mathrm{C}$, for which correlations were between .80 and .90 . At the item level, it seems that the differences between the $\mathrm{H}, \mathrm{A}$, and $\mathrm{C}$ factors are slightly obscured, causing lower consistency of primarily $\mathrm{H}$ factor across the units of analysis (subscales and items). The most robust factor was D as Table 5 shows. Moreover, the $\mathrm{D}$ factor remained the same no matter whether DELTA items were factorized with non-keyed or reverse-keyed HEXACO items. The correlation between D factors extracted in these two conditions was $(\mathrm{r}=.99)$. These factor analyses are presented in Table 6 in the Supplemental material.

\section{Discussion}


Psychosis proneness - a basic personality trait complementing the HEXACO model

The findings obtained in this study are significant concerning several aspects related to the conceptualization of the structure of basic personality traits. First, the D trait - despite being a "large package" of nine subdimensions representing a broad spectrum of psychotic-like experiences and behavioral tendencies - lies outside the HEXACO personality space as operationalized via HEXACO PI-R, 100-item version. This finding is similar to what has been found in the previous report thoroughly exploring the relationships between D and the Five-Factor Model (Knežević et al., 2017) across various assessment methods, samples, units of the analysis, and configuration of items.

Second, the level of reproducibility of the seven-factor structure of HEXACO + DELTA across the three national samples satisfies the criteria for metric invariance. Recently, support for the metric invariance of the six HEXACO factors has been obtained in a large cross-cultural study (Thielmann et al., 2019). To the best of our knowledge, this is the first study in which the crosscultural metric invariance of the seven-factor personality structure - the six HEXACO factors plus a factor capturing psychotic-like experiences - has been tested. We found that the factor loadings were identical across the three national groups. Importantly, the D facets were not an exception in terms of differential subscale functioning across national samples when compared to HEXACO facets. D was among the best-defined factors in terms of the large primary and low secondary loadings, as well as in terms of robustness across the different units of analysis (scales and items). The $\mathrm{D}$ factor turned out to be equally robust when the tendency to acquiescent responding was controlled for. The structure of the D factor remained the same irrespectively of whether its items are factorized with non-keyed or reverse-keyed (i.e. those oriented toward the psychopathological pole of a dimension) HEXACO items, the finding that is relevant for the discussion to follow. 
Psychosis proneness - a basic personality trait complementing the HEXACO model

\section{Can the separation of the Disintegration factor from the HEXACO be explained referring to} aspects other than the notion that it represents a coherent trait-like dispositional tendency?

One might speculate that $\mathrm{D}$ indices tend to form a factor because they represent maladaptive aspects of personality, while HEXACO captures normal personality variations. It implies that such a factor can be extracted only when factor analyzed with indices of normal personality. However, this seemingly plausible alternative explanation does not appear to stand up to scrutiny. For instance, when dimensions describing both normal and abnormal personality variations are factorized together a consistent recovery of six HEXACO factors plus Psychoticism has been obtained (Ashton et al., 2012; Ashton \& Lee, 2020). Such a finding is not surprising. Namely, it appears that non-psychotic maladaptive personality variations (Negative Affect, Detachment, Antagonism, and Disinhibition) represent more or less extreme aspects of behavior that easily align with the corresponding "normal" personality factors - representing mostly phenomena that occupy the opposite poles of the very same dimensions (Emotional stability, Extraversion, Honesty/Agreeableness, and Conscientiousness, respectively) (Ashton et al., 2012). Indeed, it has been argued that normal and abnormal personality variations might be represented by a single structural model (e.g., O’Connor, 2005), but also that the dimensions of a similar structural model might be even extended to incorporate other psychiatric disorders (e.g., Ruggero et al., 2019). Bearing this in mind, it does not seem likely that the inclusion of so-called maladaptive dimensions would preclude extracting the D factor similar to the one obtained here. More direct support for this expectation can be found in the data presented here: because reversekeyed HEXACO items (directed to the maladaptive poles of the traits) are similar to the measures of maladaptive personality, factorizing DELTA items together with these items should have resulted in the substantively distorted D factor comparing to the one extracted when all or only 
Psychosis proneness - a basic personality trait complementing the HEXACO model

non-keyed HEXACO items had been used. We did not find the difference in the D factor in the joint factor analysis of HEXACO and DELTA items depending on whether HEXACO items are oriented toward the pole of psychopathology or not.

However, PID-5 Psychoticism, used by Ashton and Lee (2020) is conceptualized differently from D. Namely, while PID-5 Psychoticism contains only three subdimensions based on the conceptualizations from the clinical setting (Unusual beliefs, Perceptual Distortions, and Eccentricity), D contains nine subdimensions based on a bottom-up psychometric approach. It could be argued that such a broad spectrum of phenomena covered by the D model would not form a factor if normal personality variations are factor-analyzed together with abnormal personality variations. And yet, we found that all nine D subdimensions separate from HEXACO. Our position is that there is no harm in being overinclusive in the contents ascribed to the domain. The whole package of D subdimensions should be considered as a set of hypotheses of what this domain consists of. However, these assumptions are based on good reasons - first of all empirical. These reasons had convinced the authors to exclude the dimension of Social Anhedonia (Knezevic et al., 2017) from the content of the domain. Whether they should exclude some other subdimension is a matter of evidence. The authors do not insist that Apathy/ Depression is the exclusive manifestation of D. Most likely, it is an interstitial trait, i.e., related to two or more factors, where D still has critical importance. The likely scenario - announced by the previous data - would be that some aspects of Depression - such as apathy, abulia, and self-harm are primarily induced by $\mathrm{D}$, while sadness and melancholic mood are primarily related to $\mathrm{N}$ and low X.

The authors could have pursued an alternative strategy - starting from the undeniable core contents of the domain (such as perceptual distortions and unusual beliefs), and then enlarge it if 
Psychosis proneness - a basic personality trait complementing the HEXACO model

necessary. They prefer the first one because it gives a better sense of the broadness of the domain and complexity of a possible underlying (biological) mechanism explaining the individual differences along this dimension. It appears that the PID-5 model of Psychoticism cannot accommodate the available evidence on the likely constituents of the domain. To cite only the most recent publications, evidence for the HiTOP dimensional model suggests mania be part of the domain (Kotov et al., 2017), while the evidence based on simultaneous factor analysis of NEO PI-R and various measures of psychopathology suggests paranoid-like tendencies (Mistrust) to be part of the psychoticism factor (Ashton \& Lee, 2020). This evidence is in line with the D model.

Bearing all the aforementioned in mind, it is very likely that reasons other than "psychopathology" are behind the separation of the psychotic-like manifestations of D (or other psychosis-proneness dimensions) from the manifestations of other normal and maladaptive personality dimensions. The available evidence seems to suggest that psychotic-like experiences are manifestations of a sui generis trait-like tendency, a tendency that is coherent, broader than initially expected, cross-culturally robust, and distinct from other basic traits. The authors' position is that the contents captured by $\mathrm{D}$ facets emanate from a common source, mechanism, or process defined as the proneness to relate unrelated phenomena, apophenia, or making falsepositive errors (Knežević et al., 2017). Thus, Corlett and Fletcher (2012), showed that nonclinical schizotypal experiences significantly correlate with aberrant frontal and striatal prediction of error signals. There is nothing unusual in the expectation that the system underlying generation of false-positive errors and the related illusory pattern perception is largely independent of, for example, the system generating fear (Neuroticism), or the seeking system (Openness/Extraversion), or the system generating rage (Agreeableness) (Davis \& Panksepp, 2011; Knežević et al., 2019). That is, we argue that D represents a unique and specific system that 
Psychosis proneness - a basic personality trait complementing the HEXACO model

is separate from other systems underlying other traits, not a spurious, method factor resulting from the presence of psychopathology.

Having in mind the behavioral broadness of the D domain and consequential massiveness of its neural representations (D facets capture perceptual, cognitive, emotional, somatic, and motoric phenomena), it is likely that the system causing variations in D might reflect some basic aspect of the organization/functioning of brain system as it is the case with other basic personality traits (DeYoung et al., 2012). Whether it is based on a dopaminergic neural system (Panksepp, 1998), disturbances in internal representations of contextual information (Cohen \& ServanSchreiber, 1992) later related to the integrity of NMDA receptors (Philips \& Silverstein, 2003), or some other neural mechanism remains to be empirically explored. An in-depth treatment of the other characteristics of $\mathrm{D}$ that justify its conceptualization as a personality trait - such as its adaptive potentials, i.e., possible evolutionary advantages of making false-positive errors, its normal distribution in the general population is based on the random household sample (Knezevic et al., 2017), or relationships with many aspects of socially relevant behavior/adaptations - can be found in other contributions (e.g., Medjedovic \& Knezevic, 2019, or Lazarevic et al., 2021).

\section{Limitations of the study}

One of the limitations of the present study is the absence of other methods of assessment (e.g., other observers). Specifically, the demonstrated separation of D from HEXACO factors might be an artifact of relying on a single method of assessment, i.e., self-report. The work of Knezevic et al. (2017) testing the relationship between D and the Five-Factor Model included ratings of close others (both mothers and fathers), which allowed for the control of the method of assessment. These analyses showed that the relationships between D and the FFM including only self-report measures resulted in a pattern very similar to that obtained in analyses in which the 
Psychosis proneness - a basic personality trait complementing the HEXACO model

impact of method factor(s) (i.e., variance due to the observers) was taken into account. Since there were no particular reasons to assume that the method factor would turn out to be of importance for the HEXACO but not for the FFM, we decided not to include ratings by others, but rather to include non-WEIRD samples from different nations, representative of the respective general population.

Another limitation is the use of the 20-item version of DELTA to measure D, which implies that subcomponents are measured by either 3 or 2 items. The consequence of it is the relatively low reliability of the subscales. However, since HEXACO domain scales contain 16 items, it was fair to use a version of DELTA with a comparable number of items for the factor analyses, especially those done at the item level. Anyway, it turned out that although low, the reliability of the nine DELTA subscales in the 20-item version used in this study is mostly comparable to the reliability of HEXACO subscales. Finally, our findings are highly similar to those obtained in the joint factor analysis of HEXACO PI-R (100 items version) and the full, 110 item version of D (containing reverse keyed items, Medjedovic, 2014), suggesting that the findings are generalizable across the versions of the instruments used.

To provide strong evidence in support of cross-cultural invariance of the seven-factor model, testing across more than three nations is needed. It is important to include non-European nations, i.e., those more culturally distant from the selected three nations regarding the variables that might influence personality scores. However, having in mind replicability of the HEXACO factorial structure across the non-European nations (Thielmann et al., 2019), the likelihood of obtaining different results regarding the presented seven-factor model with non-European nations is not high. "We have no reason to believe that the results depend on other characteristics of the participants, materials, or context." (Simons et al., 2017, p. 4). 
Psychosis proneness - a basic personality trait complementing the HEXACO model

\section{Conclusions}

Although capturing a broad spectrum of psychotic-like phenomena, the D factor emerged as clearly separate from the HEXACO personality factors in three samples (X, Y, Z). Empirical support was found for the metric invariance of a seven-factor personality structure consisting of the D + HEXACO factor across the three nations. D was found to be one of the best defined (coherent), and most robust factors across the national groups but also across the level of the analysis (subscales vs. items). The robustness of the D factor in the seven factor-solution was found no matter whether factor analysis was done using exclusively psychopathology-oriented (reverse keyed) or non-keyed HEXACO items. Controlling for the acquiescence in D facets did not influence the obtained seven-factor solution in any substantive manner. Consequently, the most parsimonious explanation of these findings seems to be that the nine facets reflect a real, trait-like disposition - a disposition that is not less coherent, broad, cross-culturally robust, or relevant than the other six basic personality traits.

\section{References:}

Ashton, M. C., \& Lee, K. (2007). Empirical, theoretical, and practical advantages of the HEXACO model of personality structure. Personality and Social Psychology Review, 11, 150-166. https://doi.org/10.1177/1088868306294907

Ashton, M. C., Lee, K., Marcus, B., \& De Vries, R. E. (2007). German lexical personality factors: Relations with the HEXACO model. European Journal of Personality, 21, 23-43. https://doi.org/10.1002/per.597

Ashton, M. C., \& Lee, K. (2012). Oddity, schizotypy/dissociation, and personality. Journal of Personality, 80, 113-134. https://doi.org/10.1111/j.1467-6494.2011.00735.x 
Psychosis proneness - a basic personality trait complementing the HEXACO model

Ashton, M. C., \& Lee, K. (2020). Recovering the HEXACO personality factors - and psychoticism - from variable sets assessing normal and abnormal personality. Journal of Individual Differences, 41, 68-77. https://doi.org/10.1027/1614-0001/a000305

Ashton, M. C., Lee, K., de Vries, R. E., Hendrickse, J., \& Born, M. P. (2012). The maladaptive personality traits of the Personality Inventory for DSM-5 (PID-5) in relation to the HEXACO personality factors and schizotypy/dissociation. Journal of Personality Disorders, 26, 641-659. https://doi.org/10.1521/pedi.2012.26.5.641

Bentler, P. M. (1990). Comparative fit indexes in structural models. Psychological Bulletin, 107, 238-246. https://doi.org/10.1037/0033-2909.107.2.238

Blanchard, J. J., \& Cohen, A. S. (2006). The structure of negative symptoms within schizophrenia: Implications for assessment. Schizophrenia Bulletin, 32, 238-245. https://doi.org/10.1093/schbul/sbj013

Browne, M. W. (1968). A comparison of factor analytic techniques. Psychometrika, 33, 267-334. https://doi.org/10.1007/BF02289327

Caspi, A., Houts, R. M., Belsky, D. W., Goldman-Mellor, S. J., Harrington, H., Israel, S.,... Meier, M. H. (2014). The p factor: One general psychopathology factor in the structure of psychiatric disorders? Psychological Science, 2, 119-137. https://doi.org/10.1177/2167702613497473

Chen, F. F. (2007). Sensitivity of goodness of fit indexes to lack of measurement invariance. Structural Equation Modeling, 14, 464-504. https://doi.org/10.1080/10705510701301834

Claridge, G., McCreery, C., Mason, O., Bentall, R., Boyle, G. J., Slade, P., \& Popplewell, D. (1996). The factor structure of "schizotypal" traits: A large replication study. British Journal of Clinical Psychology, 35, 103-115. https://doi.org/10.1111/j.2044-8260.1996.tb01166.x 
Psychosis proneness - a basic personality trait complementing the HEXACO model

Cohen, J. D., \& Servan-Schreiber, D. (1992). Context, cortex, and dopamine: a connectionist approach to behavior and biology in schizophrenia. Psychological Review, 99, 45-77. https://doi.org/10.1037/0033-295X.99.1.45

Corlett, P. R., \& Fletcher, P. C. (2012). The neurobiology of schizotypy: fronto-striatal prediction error signal correlates with delusion-like beliefs in healthy people. Neuropsychologia, 50, 36123620. https://doi.org/10.1016/j.neuropsychologia.2012.09.045

Davis, K. L., \& Panksepp, J. (2011). The brain's emotional foundations of human personality and the affective neuroscience personality scales. Neuroscience and Biobehavioral Reviews, 35, 1946-1958. https://doi.org/10.1016/j.neubiorev.2011.04.004

DeYoung, C. G., Hirsh, J. B., Shane, M. S., Papademetris, X., Rajeevan, N., \& Gray, J. R. (2010). Testing predictions from personality neuroscience: Brain structure and the Big Five. Psychological Science, 21, 820-828. https://doi.org/10.1177/0956797610370159

Everitt, B. S. (1975). Multivariate analysis: The need for data, and other problems. British Journal of Psychiatry, 126, 237-240. https://doi.org/10.1192/bjp.126.3.237

Eysenck, H. J. (1977). Personality and Factor Analysis: A Reply to Guilford. Psychological Bulletin, 84, 405-411. https://doi.org/10.1037/0033-2909.84.3.405

Fonseca-Pedrero, E., Debbané, M., Ortuño-Sierra, J., Chan, R., Cicero, D. C., Zhang, L. C., Brenner, C., Barkus, E., Linscott, R. J., Kwapil, T., Barrantes-Vidal, N., Cohen, A., Raine, A., Compton, M. T., Tone, E. B., Suhr, J., Muñiz, J., Fumero, A., Giakoumaki, S., Tsaousis, I., ... Jablensky, A. (2018). The structure of schizotypal personality traits: a cross-national study. Psychological Medicine, 48, 451-462. https://doi.org/10.1017/S0033291717001829

Gorsuch, R. L. (1983). Factor analysis (2nd ed.). Hillsdale, NJ: Frlbaum. 
Psychosis proneness - a basic personality trait complementing the HEXACO model

Goulding, A. (2005). Healthy schizotypy in a population of paranormal believers and experients. Personality and Individual Differences, 38, 1069-1073. https://doi.org/10.1016/j.paid.2004.07.006

Gur, R. E., Kohler, C. G., Ragland, J. D., Siegel, S. J., Lesko, K, Bilker, W. B, Gur, R. C. (2006). Flat affect in schizophrenia: relation to emotion processing and neurocognitive measures. Schizophrenia Bulletin, 32, 279-287. https://doi.org/10.1093/schbul/sbj041

Herzog, W., \& Boomsma, A. (2009). Small-sample robust estimators of noncentrality-based and incremental model fit. Structural Equation Modeling, 16, 1-27. https://doi.org/10.1080/10705510802561279

Henrich, J., Heine, S. J., \& Norenzayan, A. (2010). The weirdest people in the world? Behavioral and Brain Sciences, 33, 61-83. https://doi.org/10.1017/S0140525X0999152X

Hofstede, G., Hofstede G. J., \& Minkov, M. (2010). Cultures and organizations: Software of the mind. Revised and Expanded 3rd Edition. New York: McGraw-Hill.

Hu, L. T. \& Bentler, P. M. (1999). Cutoff Criteria for Fit Indexes in Covariance Structure Analysis: Conventional Criteria Versus New Alternatives. Structural Equation Modeling, 6, 155. https://doi.org/10.1080/10705519909540118

Jackson, M. C. (1997). Benign schizotypy? The case of spiritual experience. In G._Claridge (Ed.), Schizotypy: Relations to illness and health (pp. 227-250). New York: Oxford University Press. Kerns, J. G. (2006). Schizotypy facets, cognitive control, and emotion. Journal of Abnormal Psychology, 115, 418-427. https://doi.org/10.1037/0021-843X.115.3.418

Klein, R. A., Vianello, M., Hasselman, F., Adams, B. G., Adams, R. B., Jr., Alper, S., ... Nosek, B. A. (2018). Many labs 2: Investigating variation in replicability across sample and 
Psychosis proneness - a basic personality trait complementing the HEXACO model

setting. Advances in Methods and Practices in Psychological Science, 1, 443-490. https://doi.org/10.1177/2515245918810225

Knežević, G. \& Keller, J. (2021). Proneness to Psychotic-Like Experiences: A Neglected

Personality Correlate of Right-Wing Authoritarianism and Prejudice. Manuscript submitted to publication.

Knežević, G., Lazarević, Lj. B., Bosnjak, M., Purić, D., Petrović, B., Teovanović, P., Opačić, G., Bodroža, B. (2016). Towards a personality model encompassing a Disintegration factor separate from the Big Five traits: A meta-analysis of the empirical evidence. Personality and Individual Differences, 95, 214-222. https://doi.org/10.1016/j.paid.2016.02.044

Knežević, G., Lazarević, L. B., Purić, D., Bosnjak, M., Teovanović, P., Petrović, B., \& Opačić, G. (2019). Does Eysenck's personality model capture psychosis-proneness? A systematic review and meta-analysis. Personality and Individual Differences, 143, 155-164.

https://doi.org/10.1016/j.paid.2019.02.009

Knežević, G., Savić, D., Kutlešić, V., \& Opačić, G. (2017). Disintegration: A Reconceptualization of Psychosis Proneness as a Personality Trait Separate from the Big Five. Journal of Research in Personality, 70, 187-201. https://doi.org/10.1016/j.jrp.2017.06.001

Kotov, R., Krueger, R. F., Watson, D., Achenbach, T. M., Althoff, R. R., Bagby, R. M., et al. (2017). The Hierarchical Taxonomy of Psychopathology (HiTOP): A dimensional alternative to traditional nosologies. Journal of Abnormal Psychology, 126, 454-477. https://doi.org/10.1037/abn0000258

Krueger, R. F., Derringer, J., Markon, K. E., Watson, D., \& Skodol, A. E. (2012). Initial construction of a maladaptive personality trait model and inventory for DSM-5. Psychological Medicine, 42, 1879-1890. https://doi.org/10.1017/S0033291711002674 
Psychosis proneness - a basic personality trait complementing the HEXACO model

Kwapil, T. R., Barrantes-Vidal, N. (2014). Schizotypy: Looking Back and Moving Forward. Schizophrenia Bulletin, 41, Issue suppl_2, 366-S373. https://doi.org/10.1093/schbul/sbu186

Lazarević, L. B., Bošnjak, M., Knežević, G., Petrović, B., Purić, D., Teovanović, P., ...Bodroža, B. (2016). Disintegration as an additional trait in the psychobiological model of personality: Assessing discriminant validity via meta-analysis. Zeitschrift für Psychologie, 224, 204-215. https://doi.org/10.1027/2151-2604/a000254.

Lazarević, L., Purić, D., Teovanović, P., Lukić, P., Zupan, Z., \& Knežević, G. (2021). What drives us to be (ir)responsible for our health during the COVID-19 pandemic? The role of personality, thinking styles, and conspiracy mentality. Personality and Individual Differences, 176. https://doi.org/10.1016/j.paid.2021.110771

Lee, K., \& Ashton, M. C. (2018). Psychometric properties of the HEXACO-100. Assessment, 25, 543-556. https://doi.org/10.1177/1073191116659134

Lenzenweger, M. F., \& Dworkin, R. H. (1996). The dimensions of schizophrenia phenomenology: Not one or two, at least three, perhaps four. The British Journal of Psychiatry, 168, 432-440. DOI:10.1192/bjp.168.4.432

Lindenmayer, J. P., Brown, E., Baker, R. W., Schuh, L. M., Shao, L., Tohen, M., ... \& Stauffer, V. L. (2004). An excitement subscale of the Positive and Negative Syndrome Scale. Schizophrenia Research, 68, 331-337. https://doi.org/10.1016/S0920-9964(03)00087-2

MacCallum, R. C., Widaman, K. F., Zhang, S., \& Hong, S.(1999). Sample size in factor analysis. Psychological Methods, 4, 84-89. https://doi.org/10.1037/1082-989X.4.1.84

Marsh, H. W., Lüdtke, O., Muthén, B., Asparouhov, T., Morin, A. J. S., Trautwein, U., \& Nagengast, B. (2010). A new look at the big five factor structure through exploratory structural equation modeling. Psychological Assessment, 22, 471-491. https://doi.org/10.1037/a0019227 
Psychosis proneness - a basic personality trait complementing the HEXACO model

Medjedovic, J. (2014). Should the space of basic personality traits be extended to include the disposition toward psychotic-like experiences? Psihologija, 47, 169-184. DOI:

\subsection{8/PSI1402169M}

Medjedovic, J., Colovic, P., Dinic, B. M., \& Smederevac, S. (2017). The HEXACO personality inventory: Validation and psychometric properties in the Serbian language. Journal of Personality Assessment, 5, 1-7. https://doi.org/10.1080/00223891.2017.1370426

Međedović, J., \& Knežević, G. (2019). Dark and peculiar: The key features of militant extremist thinking pattern? Journal of Individual Differences, 40, 92-103. https://doi.org/10.1027/1614$\underline{0001 / \mathrm{a} 000280 .}$

Momirović, K. (1998). O realnoj egzistenciji psiholoških konstrukata (On the real existence of psychological constructs). In: K. Momirović (Ed.): Realnost psiholoških konstrukata (Reality of psychological constructs). Institut za psihologiju, laboratorija za eksperimentalnu psihologiju, Beograd.

Moshontz, H., Campbell, L., Ebersole, C. R., IJzerman, H., Urry, H. L., Forscher, P. S., ... \& Castille, C. M. (2018). The Psychological Science Accelerator: Advancing psychology through a distributed collaborative network. Advances in Methods and Practices in Psychological Science, 1, 501-515. https://doi.org/10.1177/2515245918797607

Muthén, L.K. and Muthén, B.O. (1998-2012). Mplus User’s Guide. Seventh Edition. Los Angeles, CA: Muthén \& Muthén.

Nettle, D. (2006). Schizotypy and mental health amongst poets, visual artists, and mathematicians. Journal of Research in Personality, 40, 876-890. https://doi.org/10.1016/j.jrp.2005.09.004

O’Connor, B. P. (2005). A search for consensus on the dimensional structure of personality disorders. Journal of Clinical Psychology, 61, 323-345. https://doi.org/10.1002/jclp.20017 
Psychosis proneness - a basic personality trait complementing the HEXACO model

Olaru, G., Witthöft, M., \& Wilhelm, O. (2015). Methods matter: testing competing models for designing short-scale big-five assessments. Journal of Research in Personality, 59, 56-68. https://doi.org/10.1016/j.jrp.2015.09.001

Panksepp, J. (1998). Affective neuroscience: The foundations of human and animal emotions. Oxford: University Press.

Philips, W. A., \& Silverstein, S. M. (2003). Convergence of biological and psychological perspectives on cognitive coordination in schizophrenia. Behavioral and Brain Sciences, 26, 65138. https://doi.org/10.1017/S0140525X03000025

Reichenberg, A. \& Harvey, P. D. (2007). Neuropsychological impairments in schizophrenia: Integration of performance-based and brain imaging findings. Psychological Bulletin, 133, 833858. https://doi.org/10.1037/0033-2909.134.3.382

Ristic, I., Maric, N., \& Knezevic, G. (2021). Do individuals diagnosed with psychosis and those from the general population share the same personality space as defined by the Big Five and Disintegration?. Manuscript in preparation.

Ronald, A. (2015). Recent quantitative genetic research on psychotic experiences: New approaches to old questions. Current Opinion in Behavioral Sciences, 2, 81-88. https://doi.org/10.1016/j.cobeha.2014.10.001

Rössler, W., Hengartner, M. P., Ajdacic-Gross, V., Haker, H., Gamma, A., \& Angst, J. (2011). Subclinical psychosis symptoms in young adults are risk factors for subsequent common mental disorders. Schizophrenia Research, 131, 18-23. https://doi.org/10.1016/j.schres.2011.06.019 Ruggero, C. J., Kotov, R., Hopwood, C. J., First, M., Clark, L. A., Skodol, A. E., ...Zimmermann, J. (2019). Integrating the Hierarchical Taxonomy of Psychopathology (HiTOP) into clinical 
Psychosis proneness - a basic personality trait complementing the HEXACO model

practice. Journal of Consulting and Clinical Psychology, 87, 1069-1084. https://doi.org/10.1037/ccp0000452

Sanislow, C. A., Little, T. D., Ansell, E. B., Grilo, C. M., Daversa, M., et al. (2009). Ten-year stability and latent structure of the DSM-IV schizotypal, borderline, avoidant, and obsessivecompulsive personality disorders. Journal of Abnormal Psychology, 118, 507-519. https://doi.org/10.1037/a0016478

Saucier, G. (2003). Factor structure of English-language personality type-nouns. Journal of Personality and Social Psychology, 85, 695-708. https://doi.org/10.1037/0022-3514.85.4.695

Serretti, A., \& Olgiati, P. (2004). Dimensions of major psychoses: a confirmatory factor analysis of six competing models. Psychiatry Research, 127, 101-109. https://doi.org/10.1016/j.psychres.2003.07.005

Simons, D. J., Shoda, Y., \& Lindsay, S. D. (2017). Constraints on Generality (COG): A Proposed Addition to All Empirical Papers. Perspectives on Psychological Science, 12, 1123-1128. https://doi.org/10.1177/1745691617708630

Stuart, G. W., Pantelis, C., Klimidis, S. \& Minas, I. H. (1999). The three-syndrome model of schizophrenia: Meta-analysis of an artefact. Schizophrenia Research, 39, 233-242. https://doi.org/10.1016/S0920-9964(99)00019-5

Thielmann, I., Akrami, N., Babarović, T., Belloch, A., Bergh, R., Chirumbolo, A., ... \& Gnisci, A. (2019). The HEXACO-100 across 16 languages: A large-scale test of measurement invariance. Journal of Personality Assessment, 1-13. https://doi.org/10.1080/00223891.2019.1614011

Watson, D., Clark, L. A., \& Chmielewski, M. (2008). Structures of personality and their relevance to psychopathology: II. Further articulation of a comprehensive unified trait structure. Journal of Personality, 76, 1545-1585. https://doi.org/10.1111/j.1467-6494.2008.00531.x 
Psychosis proneness - a basic personality trait complementing the HEXACO model

Table 1

HEXACO and DELTA descriptive statistics and reliabilities (in Z, Y, and X)

\begin{tabular}{|c|c|c|c|c|c|c|c|c|c|c|c|c|c|}
\hline & \multicolumn{4}{|c|}{$\underline{\mathrm{Z}}(\mathrm{N}=339)$} & \multicolumn{4}{|c|}{$\mathrm{Y}(\mathrm{N}=305)$} & \multicolumn{4}{|c|}{$\mathrm{X}(\mathrm{N}=367)$} & \multirow{2}{*}{$\begin{array}{l}\text { Total } \\
(\mathrm{N}=1011) \\
\text { Cronbach } \\
\alpha\end{array}$} \\
\hline & $\mathrm{M}$ & $\mathrm{SD}$ & Sk & $\mathrm{Ku}$ & $\mathrm{M}$ & SD & Sk & $\mathrm{Ku}$ & $\mathrm{M}$ & SD & Sk & $\mathrm{Ku}$ & \\
\hline Honesty & 3.58 & .59 & -.24 & .06 & 3.57 & .56 & .04 & -.28 & 3.58 & .63 & -.64 & .46 & .80 \\
\hline Sincerity & 3.50 & .80 & -.12 & -.25 & 3.38 & .70 & .14 & -.24 & 3.78 & .84 & -.68 & .28 & .61 \\
\hline Fairness & 3.67 & 1.06 & -.57 & -.46 & 3.70 & .93 & -.21 & -.88 & 3.82 & .98 & -.74 & .05 & .73 \\
\hline Greed Avoidance & 3.15 & .90 & -.15 & -.52 & 3.50 & .83 & -.27 & -.57 & 3.19 & 1.02 & -.28 & -.73 & .73 \\
\hline Modesty & 4.02 & .73 & -.45 & -.48 & 3.70 & .66 & .11 & -.59 & 3.54 & .65 & -.29 & .09 & .58 \\
\hline Emotionality & 3.25 & .62 & -.22 & -.18 & 3.20 & .49 & -.07 & .48 & 3.30 & .56 & .24 & -.15 & .79 \\
\hline Fearfulness & 3.12 & .84 & -.26 & -.48 & 2.97 & .69 & -.06 & .30 & 2.87 & .88 & .11 & -.58 & .56 \\
\hline Anxiety & 3.55 & .93 & -.29 & -.54 & 3.38 & .81 & -.13 & -.18 & 3.57 & .68 & -.23 & .07 & .63 \\
\hline Dependence & 2.91 & .80 & -.12 & -.38 & 2.97 & .67 & -.20 & .01 & 3.06 & .81 & .08 & -.52 & .58 \\
\hline Sentimentality & 3.42 & .81 & -.27 & -.20 & 3.50 & .74 & -.30 & .00 & 3.69 & .74 & -.39 & -.21 & .67 \\
\hline$\underline{\text { eXtraversion }}$ & 3.14 & .71 & .02 & .05 & 3.23 & .54 & .00 & .72 & 3.50 & .58 & -.35 & .43 & .86 \\
\hline Social Self Esteem & 3.57 & .86 & -.38 & -.43 & 3.62 & .76 & -.24 & -.37 & 3.66 & .66 & -.52 & .35 & .67 \\
\hline Social Boldness & 2.74 & .93 & .31 & -.34 & 2.94 & .72 & -.07 & .00 & 3.01 & .80 & .18 & -.48 & .65 \\
\hline Sociability & 3.05 & .91 & -.16 & -.34 & 3.13 & .76 & -.03 & .05 & 3.83 & .73 & -.41 & -.26 & .74 \\
\hline Liveliness & 3.20 & .87 & -.17 & -.49 & 3.25 & .72 & -.21 & -.14 & 3.51 & .77 & -.33 & .08 & .68 \\
\hline Agreeableness & 3.03 & .64 & -.04 & -.09 & 3.01 & .49 & -.09 & .46 & 3.05 & .53 & -.23 & -.11 & .81 \\
\hline Forgiveness & 2.51 & .79 & .15 & -.30 & 2.45 & .70 & .31 & .26 & 2.80 & .71 & -.04 & -.11 & .60 \\
\hline Gentleness & 3.43 & .73 & -.15 & -.24 & 3.30 & .62 & -.14 & .34 & 3.34 & .72 & -.21 & -.44 & .60 \\
\hline Flexibility & 3.05 & .77 & .01 & -.37 & 3.07 & .66 & -.22 & .44 & 3.00 & .76 & .09 & -.30 & .55 \\
\hline Patience & 3.13 & .94 & -.15 & -.56 & 3.23 & .69 & -.03 & -.21 & 3.08 & .75 & -.06 & -.17 & .62 \\
\hline Conscientiousness & 3.63 & .56 & -.15 & -.33 & 3.45 & .46 & .31 & .15 & 3.62 & .52 & -.33 & .18 & .79 \\
\hline Organization & 3.94 & .73 & -.54 & -.25 & 3.64 & .64 & -.23 & -.24 & 3.66 & .76 & -.64 & .37 & .57 \\
\hline Diligence & 3.63 & .79 & -.32 & -.36 & 3.54 & .66 & -.14 & -.22 & 3.86 & .68 & -.48 & .12 & .62 \\
\hline Perfectionism & 3.54 & .72 & -.04 & -.36 & 3.41 & .64 & .00 & .18 & 3.50 & .74 & -.28 & -.24 & .55 \\
\hline Prudence & 3.41 & .72 & -.11 & -.09 & 3.21 & .61 & .03 & -.06 & 3.45 & .80 & -.57 & .04 & .61 \\
\hline Openness & 3.22 & .70 & -.22 & -.21 & 2.99 & .56 & .16 & .23 & 3.37 & .67 & -.37 & -.12 & .84 \\
\hline Aesthetic App. & 3.26 & .96 & -.23 & -.40 & 2.87 & .83 & .04 & -.61 & 3.38 & .95 & -.41 & -.58 & .65 \\
\hline Inquisitiveness & 3.31 & .99 & -.37 & -.58 & 3.10 & .86 & -.08 & -.44 & 3.42 & .90 & -.42 & -.29 & .67 \\
\hline Creativity & 3.23 & .89 & -.21 & -.55 & 3.10 & .73 & -.03 & .04 & 3.51 & .87 & -.23 & -.66 & .59 \\
\hline Unconventionality & 3.09 & .67 & .10 & -.02 & 2.89 & .61 & .14 & .17 & 3.17 & .79 & -.26 & -.02 & .44 \\
\hline Disintegration & 2.33 & .80 & .42 & -.30 & 2.37 & .71 & .31 & -.60 & 2.00 & .67 & .57 & -.35 & .92 \\
\hline Gen. Cog./Exec. Imp & 2.74 & .94 & .02 & -.64 & 2.54 & .84 & .37 & -.14 & 2.19 & .94 & .61 & -.37 & .66 \\
\hline Perceptual Distort. & 2.00 & 1.02 & .79 & -.36 & 2.12 & 1.00 & .54 & -.68 & 1.64 & .78 & 1.24 & .74 & .78 \\
\hline Paranoia & 2.21 & .96 & .63 & -.16 & 2.23 & .88 & .53 & -.29 & 2.11 & .77 & .81 & 1.03 & .46 \\
\hline Apathy/Depression & 2.10 & 1.13 & .79 & -.42 & 2.05 & 1.04 & .76 & -.42 & 1.49 & .69 & 1.73 & 3.19 & .71 \\
\hline Somatoform Dysreg. & 2.19 & 1.09 & .58 & -.67 & 2.32 & 1.05 & .50 & -.57 & 1.88 & .99 & .99 & .28 & .62 \\
\hline Magical Thinking & 2.09 & .93 & .71 & -.05 & 2.19 & .88 & .55 & .08 & 1.94 & .95 & .81 & -.16 & .47 \\
\hline Enhanc. Awareness & 2.65 & 1.08 & .14 & -.88 & 2.85 & .92 & -.03 & -.36 & 2.21 & 1.12 & .64 & -.61 & .49 \\
\hline Flattened Affect & 2.35 & 1.05 & .50 & -.55 & 2.49 & 1.01 & .28 & -.70 & 2.11 & 1.10 & .82 & -.33 & .72 \\
\hline Mania & 2.61 & .92 & .11 & -.31 & 2.51 & .80 & .18 & .08 & 2.48 & .99 & .37 & -.60 & .42 \\
\hline
\end{tabular}

Note. M - Means; SD - Standard Deviation; SK - Skewness; Ku - Kurtosis. Scores for the domains and facets of both instruments were calculated as average values of the scale items. 
Psychosis proneness - a basic personality trait complementing the HEXACO model

Table 2

Invariance of the seven-factor models of 24 HEXACO + 9 DELTA facet scales evaluated by multigroup ESEM and comparison with six- and five-factor models

\begin{tabular}{|c|c|c|c|c|c|c|}
\hline Models & $\chi^{2}$ & df & $\chi^{2 / \mathrm{df}}$ & RMSEA & CFI & SRMR \\
\hline Model 1. Configural invariance & 1841.14 & 954 & 1.93 & $.053(.049-.056)$ & .923 & .028 \\
\hline $\begin{array}{l}\text { Model } 2 \text {. Configural invariance (+ two } \\
\text { error covariances) }\end{array}$ & 1730.32 & 948 & 1.82 & $.049(.046-.053)$ & .932 & .027 \\
\hline Model 3. Metric invariance & 2614.71 & 1318 & 1.98 & $.054(.051-.057)$ & .888 & .062 \\
\hline $\begin{array}{l}\text { Model 4. Metric invariance (+ two error } \\
\text { covariances) }\end{array}$ & 2455.72 & 1312 & 1.87 & $.051(.048-.054)$ & .901 & .061 \\
\hline
\end{tabular}

\begin{tabular}{|c|c|c|c|c|c|c|}
\hline Model 4a. For A domain & 2534.82 & 1320 & 1.92 & $.052(.049-.055)$ & .895 & .063 \\
\hline Model 4b. For E domain & 2508.82 & 1320 & 1.91 & $.052(.049-.055)$ & .897 & .063 \\
\hline Model 4c. For O domain & 2532.57 & 1320 & 1,92 & $.052(.049-.055)$ & .895 & .063 \\
\hline Model 4d. For C domain & 2554.29 & 1320 & 1.94 & $.053(.050-.056)$ & .893 & .064 \\
\hline Model 4e. For D domain & 2649.53 & 1330 & 1.99 & $.054(.051-.057)$ & .886 & .069 \\
\hline Model 4f. For H domain & 2641.36 & 1320 & 2.00 & $.054(.051-.058)$ & .885 & .067 \\
\hline Model 4g. For X domain & 2670.95 & 1320 & 2.03 & $.055(.052-.058)$ & .883 & .070 \\
\hline $\begin{array}{l}\text { Model 5. Scalar invariance (+ two error } \\
\text { covariances) }\end{array}$ & 3354.21 & 1378 & 2.43 & $.065(.062-.068)$ & .829 & .086 \\
\hline $\begin{array}{l}\text { Model 6. Six factor model, metric } \\
\text { invariance (+ two error covariances) }\end{array}$ & 2743.86 & 1353 & 2.03 & $.055(.052-.058)$ & .879 & .067 \\
\hline $\begin{array}{l}\text { Model 7. Five-factor model, metric } \\
\text { invariance (+ two error covariances) }\end{array}$ & 3389.72 & 1393 & 2.44 & $.065(.062-.068)$ & .827 & .075 \\
\hline \multicolumn{7}{|c|}{ Note. Nested models are: Model 7 within Model 6; Model 6 within model 4; Model 4 within } \\
\hline \multirow{2}{*}{\multicolumn{7}{|c|}{$\begin{array}{l}\text { Model 2; Model } 5 \text { within Model 4; Models 4a-4g within Model 4; Model } 3 \text { within Model 4; } \\
\text { Model } 3 \text { within Model 1; Model } 1 \text { within Model 2. Error covariances were allowed between }\end{array}$}} \\
\hline & & & & & & \\
\hline \multicolumn{7}{|c|}{ Sociability and Social Boldness, and Greed Avoidance and Modesty; All $\chi^{2}$ differences between } \\
\hline
\end{tabular}


Psychosis proneness - a basic personality trait complementing the HEXACO model

Table 3

ESEM analysis of 24 HEXACO + 9 DELTA facet scales: loadings and factor intercorrelations (completely standardized solution), Model 4 from Table 2

\begin{tabular}{|c|c|c|c|c|c|c|c|}
\hline & $\mathrm{D}$ & $X$ & $\mathrm{H}$ & $\mathrm{E}$ & $\mathrm{O}$ & A & $\mathrm{C}$ \\
\hline \multicolumn{8}{|l|}{ D-Disintegration } \\
\hline $\begin{array}{l}\text { General Executive/Cognitive } \\
\text { Impairment }\end{array}$ & .75 & -.04 & .03 & .16 & -.06 & -.03 & .00 \\
\hline Perceptual Distortion & .86 & -.03 & -.06 & -.03 & .01 & .09 & .00 \\
\hline Paranoia & .74 & -.04 & .08 & -.13 & -.02 & -.11 & -.02 \\
\hline Apathy/Depression & .60 & -.35 & -.14 & -.03 & .00 & .13 & -.01 \\
\hline Somatoform Dysregulation & .79 & -.10 & -.07 & .10 & -.01 & .06 & .03 \\
\hline Magical Thinking & .81 & .21 & .13 & -.03 & .07 & -.03 & -.09 \\
\hline Enhanced Awareness & .68 & .16 & -.01 & .18 & .16 & -.01 & .09 \\
\hline Flattened Affect & .79 & -.06 & .00 & -.29 & -.03 & -.02 & .01 \\
\hline Mania & .67 & .15 & -.02 & .05 & -.10 & -.13 & -.05 \\
\hline \multicolumn{8}{|l|}{ H-Honesty/Humility } \\
\hline Sincerity & -.02 & -.03 & .33 & -.10 & .03 & .10 & .18 \\
\hline Fairness & -.07 & .02 & .64 & .03 & .04 & .08 & .10 \\
\hline Greed Avoidance & .01 & -.04 & .53 & -.12 & .00 & .04 & -.01 \\
\hline Modesty & -.23 & -.21 & .16 & .15 & -.05 & .22 & .08 \\
\hline \multicolumn{8}{|l|}{ E-Emotionality } \\
\hline Fearfulness & -.02 & -.30 & .09 & .47 & -.07 & -.03 & -.07 \\
\hline Anxiety & .14 & -.47 & -.01 & .46 & -.02 & -.13 & .25 \\
\hline Dependence & .01 & .00 & -.05 & .64 & .04 & -.02 & -.15 \\
\hline Sentimentality & -.09 & -.03 & .04 & .80 & .17 & .04 & .06 \\
\hline \multicolumn{8}{|l|}{ X-Extraversion } \\
\hline Social Self-Esteem & -.34 & .59 & .00 & -.01 & -.06 & .09 & .07 \\
\hline Social Boldness & -.01 & .56 & -.14 & -.10 & .13 & .01 & .07 \\
\hline Sociability & .02 & .57 & -.07 & .21 & -.02 & .14 & .00 \\
\hline Liveliness & -.20 & .71 & .04 & .00 & .00 & .03 & .03 \\
\hline \multicolumn{8}{|l|}{$\underline{\mathrm{A}-\text { Agreeableness }}$} \\
\hline a Forgiveness & .08 & .22 & .07 & -.03 & .12 & .49 & -.16 \\
\hline Gentleness & .08 & .16 & .05 & .16 & -.05 & .64 & .03 \\
\hline Flexibility & -.14 & .02 & .06 & .02 & -.02 & .69 & -.03 \\
\hline Patience & -.04 & .13 & .03 & -.15 & .08 & .58 & .02 \\
\hline \multicolumn{8}{|l|}{ C-Conscientiousness } \\
\hline Organization & -.15 & .14 & .08 & .06 & -.11 & .02 & .56 \\
\hline Diligence & -.03 & .31 & .00 & -.02 & .16 & .01 & .56 \\
\hline Perfectionism & .09 & .08 & .06 & .06 & .14 & -.14 & .67 \\
\hline Prudence & -.26 & -.08 & .06 & -.21 & .02 & .17 & .47 \\
\hline
\end{tabular}


Psychosis proneness - a basic personality trait complementing the HEXACO model

\begin{tabular}{|c|c|c|c|c|c|c|c|}
\hline \multicolumn{8}{|l|}{ O-Openness } \\
\hline Aesthetic Appreciation & .01 & -.06 & .12 & .03 & .75 & .05 & -.02 \\
\hline Inquisitiveness & -.08 & .01 & -.04 & -.08 & .64 & .03 & .10 \\
\hline Creativity & .02 & .10 & .06 & .06 & .74 & -.07 & .01 \\
\hline Unconventionality & .06 & .02 & -.20 & .00 & .70 & .00 & -.07 \\
\hline$\underline{Z}$ sample & $\mathrm{D}$ & $\mathrm{X}$ & $\mathrm{H}$ & $\mathrm{E}$ & $\mathrm{O}$ & A & \\
\hline $\mathrm{X}$ & -.36 & & & & & & \\
\hline $\mathrm{H}$ & -.43 & .02 & & & & & \\
\hline $\mathrm{E}$ & .11 & -.05 & .33 & & & & \\
\hline $\mathrm{O}$ & .03 & .36 & .15 & -.03 & & & \\
\hline A & -.28 & .38 & .37 & -.14 & .27 & & \\
\hline $\mathrm{C}$ & -.28 & .24 & .30 & .15 & .25 & .12 & \\
\hline \multicolumn{8}{|l|}{ Y sample } \\
\hline $\mathrm{X}$ & -.32 & & & & & & \\
\hline $\mathrm{H}$ & -.46 & .15 & & & & & \\
\hline $\mathrm{E}$ & .10 & .08 & .00 & & & & \\
\hline $\mathrm{O}$ & .09 & .34 & -.12 & .10 & & & \\
\hline A & -.14 & .09 & .13 & .06 & .24 & & \\
\hline $\mathrm{C}$ & -.16 & -.06 & .15 & .27 & .14 & .13 & \\
\hline \multicolumn{8}{|l|}{ X sample } \\
\hline $\mathrm{X}$ & -.07 & & & & & & \\
\hline $\mathrm{H}$ & .06 & -.13 & & & & & \\
\hline $\mathrm{E}$ & .37 & -.07 & .28 & & & & \\
\hline $\mathrm{O}$ & -.04 & .08 & .03 & -.11 & & & \\
\hline A & -.36 & -.17 & .27 & -.23 & .01 & & \\
\hline $\mathrm{C}$ & -.06 & .27 & .09 & -.11 & .20 & .14 & \\
\hline
\end{tabular}

Note. Loadings and correlations above .30 are given in boldface 